\title{
Effect of evaporation temperature on the formation of particulate membranes from crystalline polymers by dry-cast process
}

\author{
Tai-Horng Young *, Jhi-Hsien Huang, Wen-Yuan Chuang \\ Institute of Biomedical Engineering, College of Medicine and College of Engineering, National Taiwan University, \\ Taipei 10016, Taiwan, ROC
}

Received 9 February 2001; received in revised form 18 May 2001; accepted 13 July 2001

\begin{abstract}
The membrane formation of crystalline poly(ethylene-co-vinyl alcohol) (EVAL), poly(vinylidene fluoride) (PVDF), and polyamide (Nylon-66) membranes prepared by dry-cast process was studied. Membrane morphologies from crystalline polymers were found to be strongly dependent on the evaporation temperature. At low temperatures, all the casting solution evaporated into a particulate morphology that was governed by the polymer crystallization mechanism. The rise in the evaporation temperature changed EVAL membrane structure from a particulate to a dense morphology. However, as the temperature increased PVDF and Nylon-66 membranes still exhibited particulate morphologies. The membrane structures obtained were discussed in terms of the characteristics of polymer crystallization in the casting solution theoretically. At elevated temperatures the crystallization was restricted for the EVAL membrane because the increase rate in the polymer concentration was fast relative to the time necessary for growth of nuclei. Nonetheless, the time available for PVDF and Nylon-66 with stronger crystalline properties was large enough to form the crystallizationcontrolled particulate structure that differed in particle size only. In addition, particles in the PVDF membrane were driven together to disappear the boundary, but those in the Nylon-66 membrane exhibited features of linear grain boundary. The difference in particle morphology was attributed to the Nylon-66 with the most strongly crystalline property. Therefore, the kinetic difference in the crystallization rate of the polymer solution play an important role in dominating the membrane structure by dry-cast process. (c) 2001 Elsevier Science Ltd. All rights reserved.
\end{abstract}

Keywords: Dry process; Crystalline polymers; Evaporation temperature; Membrane morphology

\section{Introduction}

Over a few decades, polymeric membranes have achieved commercial importance in a variety of separation applications. Many polymeric membranes are fabricated via a process referred to by Kesting as phase inversion [1]. The dry-cast process is one of several processes by which a casting solution is evaporated to

\footnotetext{
${ }^{*}$ Corresponding author. Tel.: +886-2-23970800, ext. 1455; fax: +886-2-23940049.

E-mail address: thyoung@ha.mc.ntu.edu.tw (T.-H. Young).
}

promote phase inversion. Although membranes prepared by dry-cast process have been used for many years in a large number of applications [1], it is quite surprising that the role of crystalline polymers in relation to membrane formation in the dry process has not yet been elucidated in detail $[2,3]$. Most of the examples, however, deal with amorphous systems $[1,4]$. Therefore, the purpose of this paper is to examine whether crystallization induced by evaporation of solvent may result in a change of the membrane structure.

The effect of evaporation temperature on the structures of the crystalline polymer membranes prepared by dry-cast process was studied. Although the dry-cast 
process is a simple experimental method, it was found that the slight temperature change could affect the membrane morphology to a great degree. There are several models proposed to describe the solvent evaporation pertinent to the dry process [5-7], but their principal research efforts are dedicated to amorphous polymers. These theories are not satisfactory in explaining the formation of membranes from crystallizable polymers. In this work, the membrane structures obtained were discussed in terms of the sequence and mechanism of phase transformations in the evaporated casting solution theoretically. It was shown that both polymer crystallization and vitrification could interfere in the membrane structure during the solvent evaporation process. After elimination of the solvent, a supersaturated solution has the opportunity for crystallization of regular segments of polymer to bring particulate feature to the morphology of the membrane [8-12]. In this way, the crystallization-controlled particulate morphology is different from a typical amorphous membrane based on the vitrification of polymer solution. Conversely, crystallization process will be hindered by the limitations of local chain motion when the solution viscosity is increased. Therefore, the basic principle of controlling membrane structures from a crystallizable polymer is the competition between crystallization and vitrification, and the evaporation rate of solvent is crucial for determining the final membrane structure.

For a given polymer system, the membrane morphology determines the transport properties and therefore dictates the separation application. In our laboratory, we have prepared crystalline polymer membranes characterized by a packed bed of nearly equal-diameter particles by directly immersing the polymer solution into a 1 -octanol bath $[13,14]$. Such particulate membranes have proved useful in microfiltration [13], plasma protein separation [14], and cell culture [15]. However, a potential problem associated with the use of 1-octanol as nonsolvent is their low solubility in water that exacerbates its subsequent extraction. Indeed, the removal of 1-octanol from the membrane required extensively washing by a series of washing steps. Therefore, to employ the dry-cast process to prepare membranes is a possible means of circumventing problems associated with the use of 1-octanol as nonsolvent.

\section{Experimental}

\subsection{Materials}

The crystalline polymer materials used in this study included poly(ethylene-co-vinyl alcohol) (EVAL, E105A containing $\approx 56 \mathrm{~mol} \%$ vinyl alcohol, Kuraray, Japan), poly(vinylidene fluoride) (PVDF, Kynar 740, Elf Ato Chem) and polyamide (Nylon-66, Zytel 101 DuPont).
Dimethyl sulfoxide, $N, N$-dimethylformamide and formic acid were purchased from Nacalai Tesque (Kyoto, Japan, extra pure reagent grade) and used as the solvent for EVAL, Nylon-66 and PVDF, respectively. Water was double distilled and deionized before use.

\subsection{Membrane preparation and characterization}

An appropriate amount of polymer was dissolved in solvent to form a $25 \mathrm{wt} . \%$ of polymer solution. The polymer solution was spread on a glass plate in a uniform thickness of $100 \mu \mathrm{m}$ by an autocoater (KCC303, RK Print-Coat Instruments, UK) at $25{ }^{\circ} \mathrm{C}$. Solvent evaporation was then permitted in an oven at different temperatures until the casting solution became a solid membrane. The membrane was then soaked in water to remove any residual solvent and removed from the glass plate.

The morphology of the membrane was examined using a scanning electron microscope (SEM). The freezedried samples were sputtered with gold and palladium in a vacuum and using a Hitachi S-800 microscope at $20 \mathrm{kV}$.

The crystalline characteristics of each membrane was studied using a sensitive DSC 2010 (TA instruments Ltd., USA) under a nitrogen atmosphere. The DSC was calibrated by using indium as standards. The temperature was raised from room temperature at a heating rate of $10{ }^{\circ} \mathrm{C} / \mathrm{min}$ to observe the endothermic peak of calorimetric transition. The melting temperature $T_{\mathrm{m}}$ is taken as the temperature at the maximum of the melting endotherm.

\section{Results and discussion}

3.1. Membranes prepared from the system poly(ethyleneco-vinyl alcohol)-dimethyl sulfoxide

Fig. 1 shows the membrane structure for EVAL solution evaporated at $35{ }^{\circ} \mathrm{C}$, where the so-called "particulate" morphology typical of that resulting from the phase inversion of EVAL solution immersed in 1-octanol as reported in our previous studies $[13,14]$ is shown. The dry-cast process is characterized by the evaporation of solvent from a homogeneous polymer solution. Generally, the complete evaporation of a polymer solution only consisting of a polymer and a solvent will result in a dense or nearly dense membrane. However, the formed membrane appears looser and there exists a number of holes surrounding the particles interconnected into continuous yet tortuous channels. It can be seen that there is little evidence of a regular cellular pore structure that is initiated by the process of liquid-liquid demixing [4]. A detail of the cross-sectional structure 

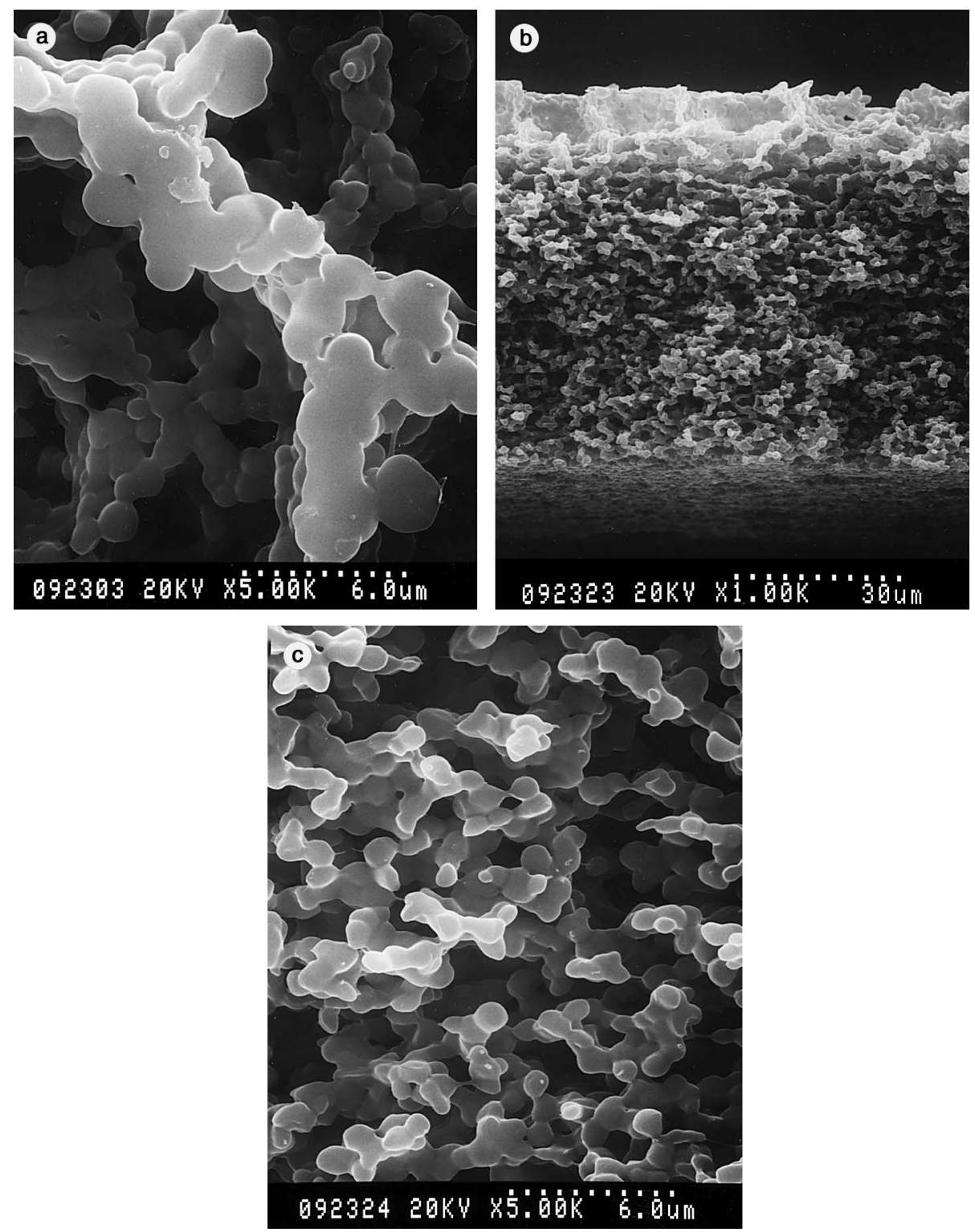

Fig. 1. SEM photomicrographs of membrane from a $25 \mathrm{wt} . \%$ of EVAL solution prepared at $35{ }^{\circ} \mathrm{C}$ of evaporation temperature. (a) Top surface; (b) cross-section and (c) magnification of (b).

shown in Fig. 1(c) shows the building spherical particles have an averaged diameter of $0.5 \mu \mathrm{m}$ of approximately equal size. This suggests all of the particles emerge from individual EVAL nuclei born roughly at the same time and affirms the domination of crystallization during the membrane formation process.

Fig. 2 shows the obtained EVAL membrane structure when the evaporation temperature in the oven was $60^{\circ} \mathrm{C}$. The membrane appears fairly dense structure without any particles or holes existing in the top surface and cross-section. By comparing Figs. 1 and 2 it is shown that particles will disappear when the evaporation temperature is increased. It implies that at least two different types of membranes can be prepared, which quite naturally suggests the formation mechanisms of these two membranes are totally different in the dry-cast process.

\subsection{Membranes prepared from the system poly (vinylidene fluoride)-dimethyl formamide}

In contrast to the dense morphology obtained for the EVAL solution evaporated at $60{ }^{\circ} \mathrm{C}, \mathrm{PVDF}$ membranes 

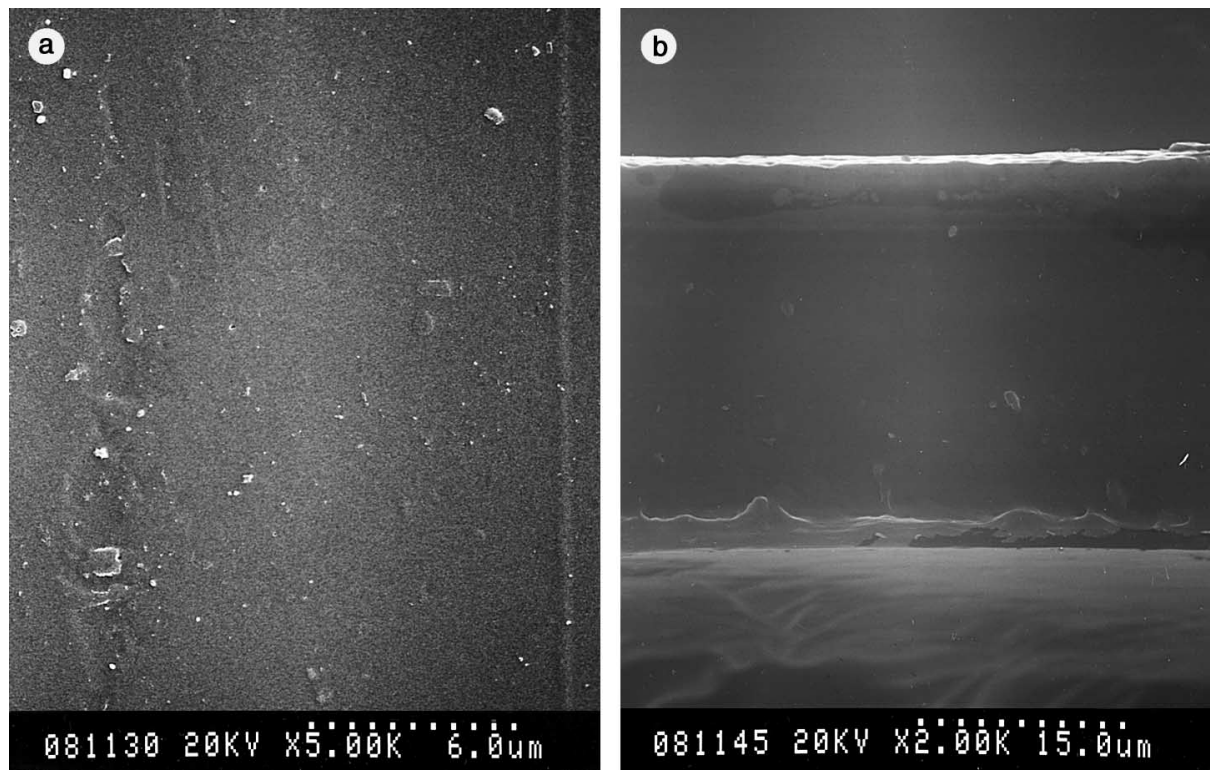

Fig. 2. SEM photomicrographs of membrane from a $25 \mathrm{wt} . \%$ of EVAL solution prepared at $60{ }^{\circ} \mathrm{C}$ of evaporation temperature. (a) Top surface and (b) cross-section.
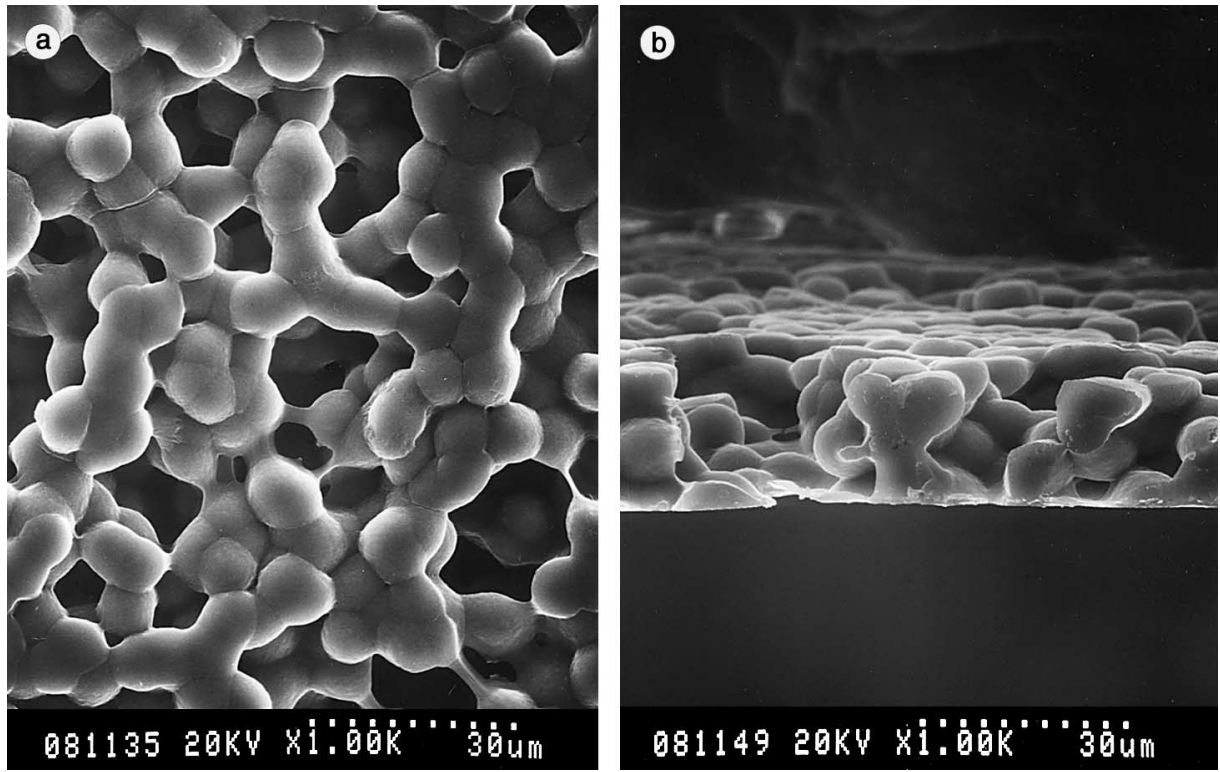

Fig. 3. SEM photomicrographs of membrane from a $25 \mathrm{wt} . \%$ of PVDF solution prepared at $60{ }^{\circ} \mathrm{C}$ of evaporation temperature. (a) Top surface and (b) cross-section.

prepared by evaporating solvent at $60{ }^{\circ} \mathrm{C}$ are characterized by the particulate morphology, as shown in Fig. 3. This PVDF membrane contains the same structural characteristics as that obtained with EVAL evaporated at $35^{\circ} \mathrm{C}$. Globular particles are distributed uniformly in this membrane. However, the sizes of these building particles are several times larger than those at EVAL membrane and fall in the range of micron order. As the evaporation temperature was increased from 60 to $150{ }^{\circ} \mathrm{C}$, the top surface of the membrane contains very small pores (Fig. 4(a)) and its cross-section likely exhibits a dense morphology similar to that obtained with 

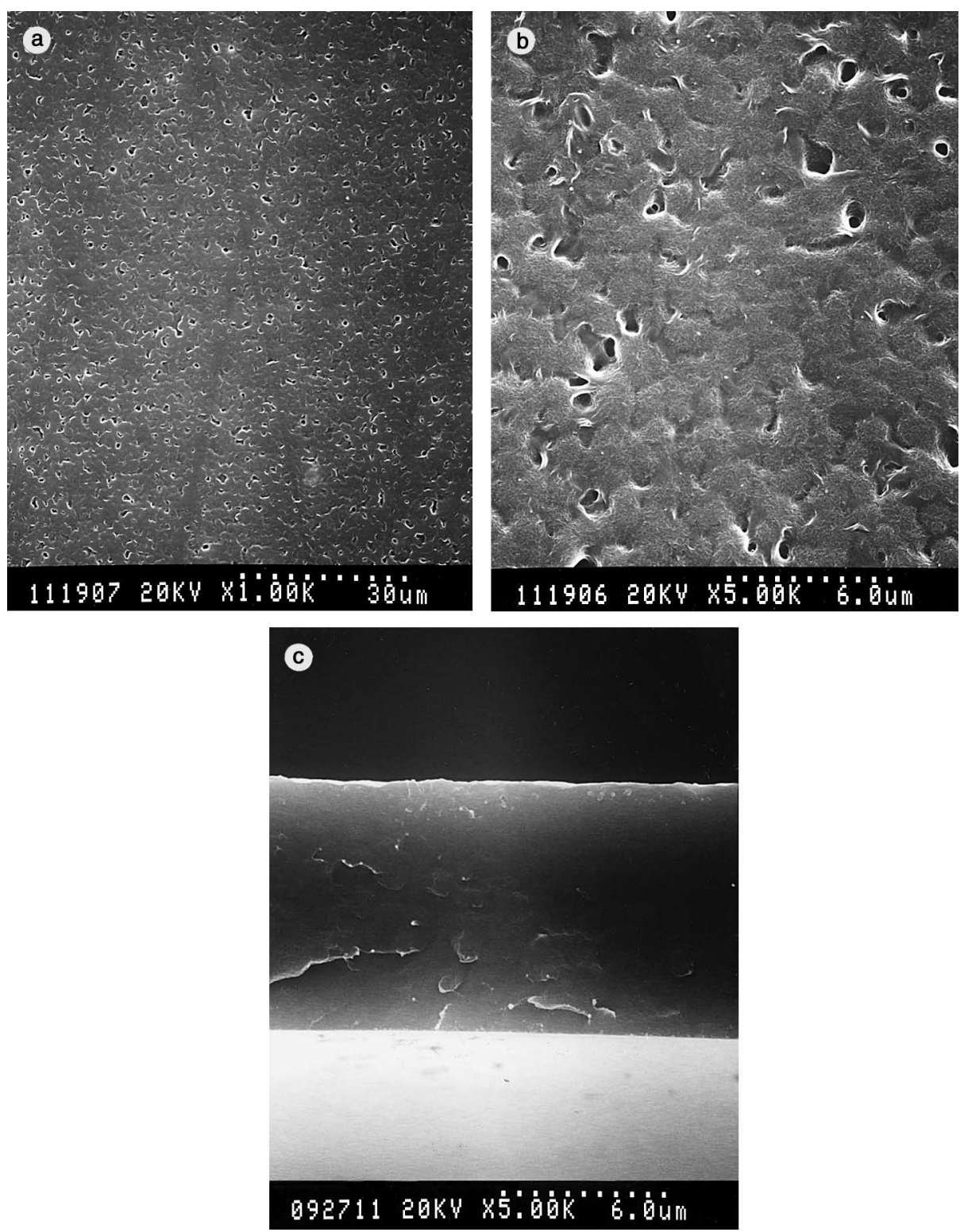

Fig. 4. SEM photomicrographs of membrane from a $25 \mathrm{wt} . \%$ of PVDF solution prepared at $150{ }^{\circ} \mathrm{C}$ of evaporation temperature. (a) Top surface; (b) magnification of (a) and (c) cross-section.

EVAL evaporated at $60{ }^{\circ} \mathrm{C}$ (Fig. 4(c)). There is almost not any particles existing in this membrane. However, it can be noticed from a larger magnification of photo that superficial small pores exist at the membrane surface, which are the spaces resulting from incomplete coalescence of particles (Fig. 4(b)). Although the particles are difficult to distinguish each other, the size of the particles is approximately $0.5-1 \mu \mathrm{m}$. By comparing Figs. 3 and 4 it is shown that particles become smaller and will be somehow driven together to disappear the boundary when the evaporation temperature is increased.
3.3. Membranes prepared from the system Nylon-66formic acid

Similar to PVDF membranes, Nylon-66 membranes prepared by evaporating solvent at $60^{\circ} \mathrm{C}$ are composed of large particles of $10 \mu \mathrm{m}$ in diameter, as shown in Fig. 5. However, the particles did not disappear even when the evaporation temperature was increased to higher than the boiling point of formic acid. The effect of the evaporation temperature on the Nylon-66 membrane morphology can be clearly observed by electron 

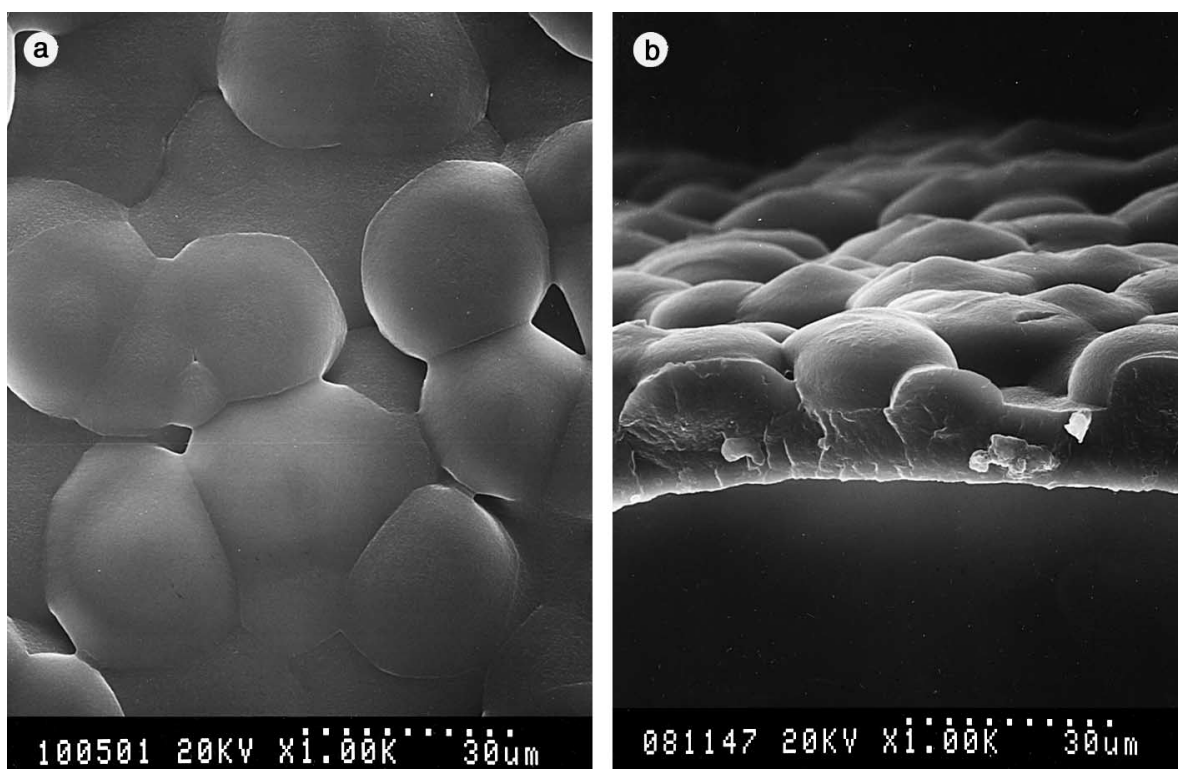

Fig. 5. SEM photomicrographs of membrane from a $25 \mathrm{wt} . \%$ of Nylon-66 solution prepared at $60{ }^{\circ} \mathrm{C}$ of evaporation temperature. (a) Top surface and (b) cross-section.

microscopy (Fig. 6). In order to confirm the dense Nylon-66 membrane could not be obtained, the evaporation temperature was increased to $250{ }^{\circ} \mathrm{C}$. The particulate characteristics of the membranes were hardly influenced by the evaporation temperature. These membranes exhibited a structure formed of package of spherical particles. In addition, particles in the PVDF membrane can coalesce into a macroscopic, dense-like structure prepared for higher evaporation temperature. This, however, does not occur in the Nylon-66 membrane. The Nylon-66 particles grow until their fronts impinge each other to form the polygonal structure with linear grain boundaries. However, similar to the particulate morphology obtained for the PVDF polymer, the size of particles decreased for the Nylon-66 membranes obtained as the evaporation temperature is increased. This indicates that the number of particle-nuclei was increased at higher evaporation temperature.

\subsection{Thermal analysis}

In order to gain insight into the role crystallization plays in determining the final membrane morphology, thermal analysis by the first heat DSC scan was done over the prepared membranes. The relatively large breadth of DSC peaks was observed for all EVAL, PVDF and Nylon-66 membranes (not shown here). Values of melting temperature and endotherm are illustrated in Table 1. Although the variation of melting temperature shows no discernible quantitative trend, the endotherms appear to decrease with increasing evapo- ration temperature irrespective of polymer. This data suggests increasing evaporation temperature may depress polymer crystallization, especially for evaporating EVAL solution changed from 35 to $60{ }^{\circ} \mathrm{C}$.

\subsection{Membrane formation mechanism}

This paper reports a study of the preparation of membranes from crystalline polymers by dry-cast process. The phase inversion is induced by the evaporation of solvent from the casting solution. Before evaporation of the solvent, polymer is dissolved in solvent to form a homogeneous solution in which the polymer molecule relaxes and behaves like an expanded coil. As solvent is evaporated from this solution, the polymer concentration is increased to reduce the solvation character of polymer in solvent. Finally, polymer coils collapse and chains entangle to gel the casting solution and then to vitrify the membrane structure. For crystalline polymers, when the solution becomes supersaturated with respect to crystallization, they can crystallize from the solution, in which molecules are folded back and forth on themselves. Therefore, during evaporation of the solvent, gelation of a crystalline polymer solution can occur by crystallization or vitrification. In other words, these two different phase inversions can compete with each other during membrane formation to influence the membrane structure.

Such a competition can be understood through the corresponding temperature-concentration diagram, as illustrated in Fig. 7 for a solution of a crystallizable 

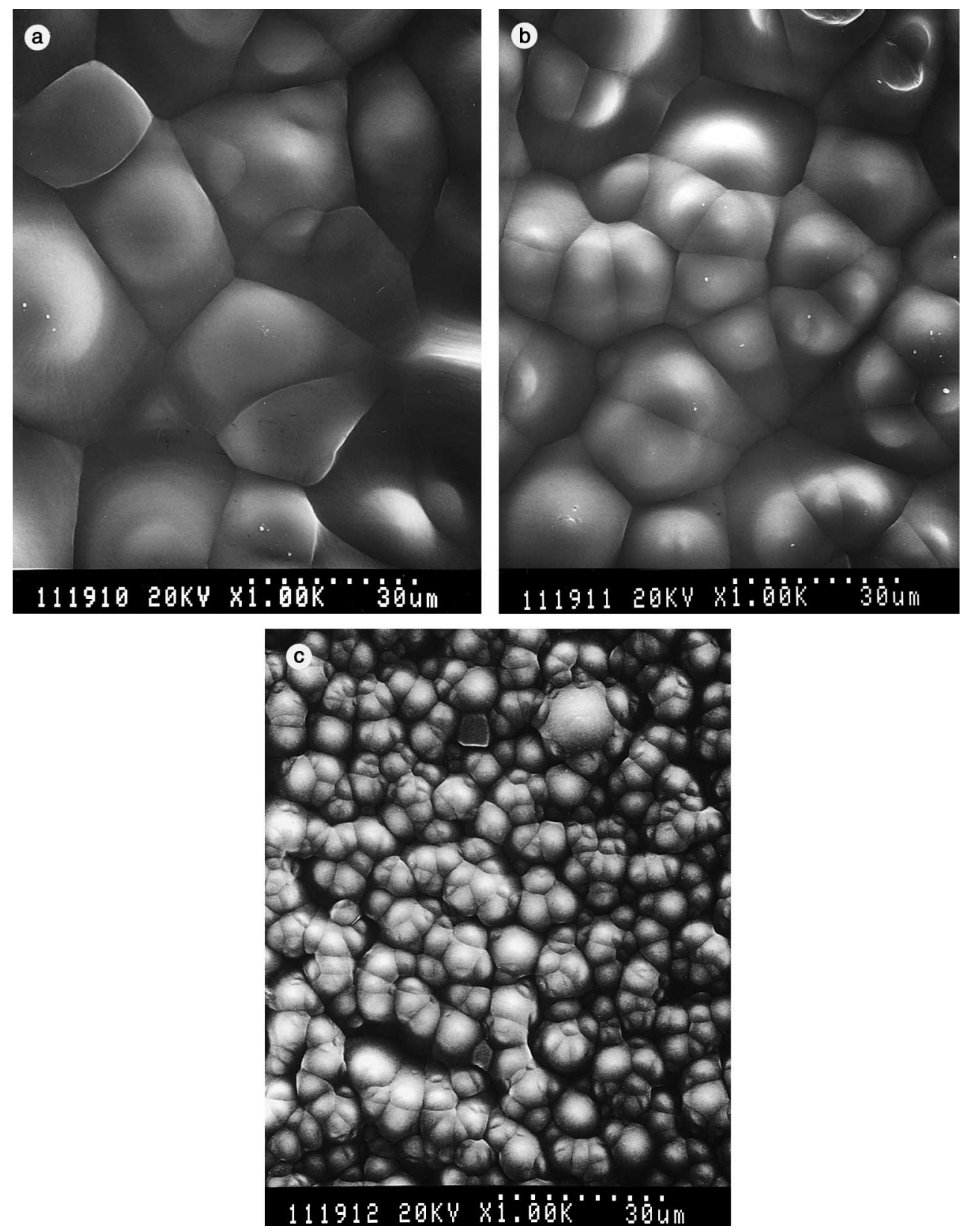

Fig. 6. SEM photomicrographs of membrane surface from a $25 \mathrm{wt} . \%$ of Nylon-66 solution prepared at different evaporation temperatures. (a) $180{ }^{\circ} \mathrm{C}$; (b) $200{ }^{\circ} \mathrm{C}$ and (c) $250{ }^{\circ} \mathrm{C}$.

polymer. The system shows upper critical solution temperature behavior and the binodal is below the crystallization line. On heating to evaporate a casting solution with composition $X$, the crystallization line can be crossed. The crystallization line in the phase diagram represents an equilibrium state only. The transition from a randomly coiled configuration in the liquid state to a three-dimension ordered configuration in the solid state is a kinetically hindered process. In general, crystallization is a slow process because of the time needed for orientation of the polymer molecule, both for nucleus formation and for growth. It is possible that the induction time is not enough for crystal nuclei to be formed or to grow when the composition change in the membrane formation is too rapid. This suggests other types of phase inversion may also play a role in the 
Table 1

DSC first heat data of EVAL, PVDF and Nylon-66 membranes

\begin{tabular}{lcll}
\hline Membrane & $\begin{array}{l}\text { Evaporation } \\
\text { temperature }\left({ }^{\circ} \mathrm{C}\right)\end{array}$ & $\begin{array}{l}\text { Melting tempera- } \\
\text { ture }\left({ }^{\circ} \mathrm{C}\right)\end{array}$ & $\begin{array}{l}\text { Heat } \\
(\mathrm{J} / \mathrm{g})\end{array}$ \\
\hline EVAL & 35 & 155.03 & 75.89 \\
EVAL & 60 & 165.98 & 55.61 \\
PVDF & 60 & 169.13 & 64.95 \\
PVDF & 150 & 171.42 & 52.29 \\
Nylon-66 & 60 & 264.25 & 68.01 \\
Nylon-66 & 180 & 261.41 & 63.56 \\
Nylon-66 & 200 & 263.59 & 61.12 \\
Nylon-66 & 250 & 263.48 & 60.46 \\
\hline
\end{tabular}

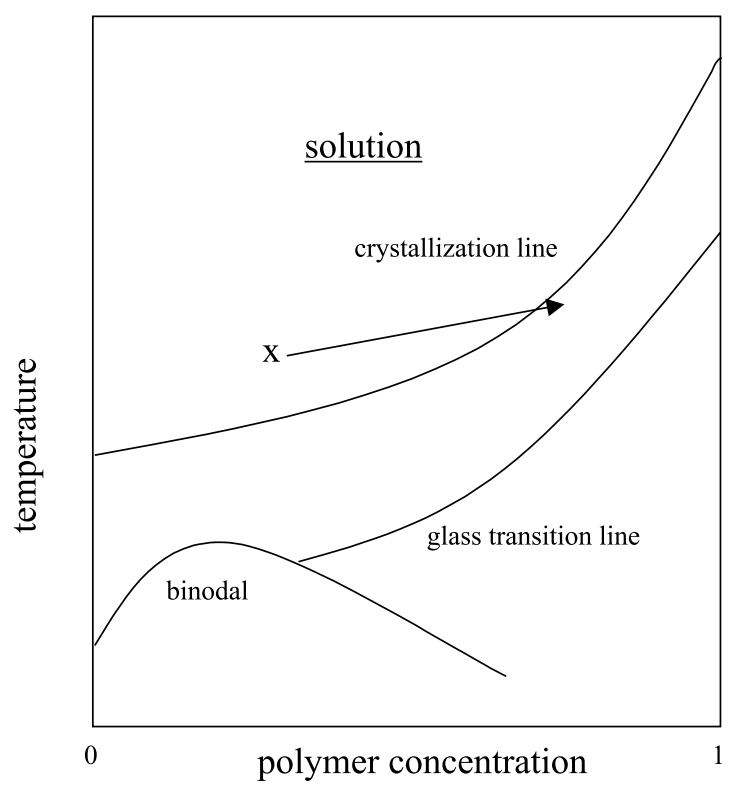

Fig. 7. Schematic representation of temperature-concentration diagram for the system crystalline polymer/solvent.

membrane formation process and such phase inversion quite naturally suggests the likelihood of chain entanglement by the system crossing some sort of viscosity boundary at much higher polymer concentrations. Glass transition depression can provide a simple explanation for the hypothesized viscosity boundary, thus leaving the vitrification process as a logical source for the dense membrane formation. Consequently, upon rapid elimination of solvent, polymer molecules may directly vitrify after the glass transition line is crossed. In this situation, the membrane structure from crystallizable polymers is the same as that from amorphous ones.

Based on the above discussion, the dry-cast system is not in thermodynamic equilibrium but is supposed to be toward the expected glass transition-concentration curve. Two opposing effects will be operative when the polymer concentration is increased: the driving force for crystallization increases with increasing the degree of supersaturation, and the reduction of chain mobility in the high polymer concentration state limiting any further evolution of the system. Thus, the membrane morphology from a crystalline polymer will not only be the result of the polymer-solvent phase behavior, but will depend to a large extent on the experimental conditions and the crystalline characteristics of the polymer. Especially, the time that is given to the system before it is frozen by vitrification of polymer is important. On evaporating the casting solution at lower temperatures, the increasing rate of polymer concentration is slower and the polymer has a longer period to become vitrified, thus nucleation is probably to occur to result in a crystallization-controlled particulate structure. The particulate morphology was indeed observed for the EVAL membrane prepared with evaporation temperature of $35{ }^{\circ} \mathrm{C}$ (Fig. 1). On the other hand, the evaporation rate of solvent was fast relative to the time necessary for EVAL crystallization at $60{ }^{\circ} \mathrm{C}$ of evaporation temperature. This means a rapidly increased EVAL concentration brings this casting solution into a state to surpass the crystallization region to preclude the possibility of any nucleation or to impede its growth only. Since the EVAL membrane evaporated at $60{ }^{\circ} \mathrm{C}$ with lower crystallinity was observed (Table 1), we consider particle nuclei could be created when solution viscosity was increased, while growth of particles was constrained. It can be expected that the dimension of nuclei is under the resolution of SEM, thus such a membrane exhibited a dense morphology (Fig. 2). Consequently, crystallization only has a small effect on the overall morphology of the formed membrane.

Contrary to the EVAL membrane, the Nylon-66 membrane evaporated at $60{ }^{\circ} \mathrm{C}$ showed a particulate structure (Fig. 5). This indicates the time available for the Nylon-66 molecules moving into successive positions in the crystal lattice at this evaporation temperature was enough. Even though the evaporation temperature was increased to $250{ }^{\circ} \mathrm{C}$, Nylon-66 membranes exhibited a particulate morphology with particles that differed in size only (Fig. 6). The observation of evaporation temperature-independent particulate morphology for Nylon-66 membranes suggests the characteristics of the system itself also effect the final result significantly. Therefore, similar to the dense EVAL membrane structure will never be realized when strongly crystalline Nylon-66 is involved.

Furthermore, the degree of supersaturation, necessary to form crystal nuclei, is measured as the distance right to the crystallization line. Applied to the dry-cast process, the degree of supersaturation increases with increasing the evaporation temperature that leads to a rapider increment of polymer concentration. As a result, more particles are nucleated per unit volume at higher evaporation temperature. The larger the nucleation 
density, the less particles are able to grow freely before they reach a neighboring particle. Therefore, the size of the particles in the Nylon-66 membranes apparently decreased with increasing evaporation temperature (Fig. 6). This result appears to be consistent with the cases of thermal induced crystallization processes in which the amount of nuclei is increased as the degree of supercooling is increased.

Similar to strongly crystalline Nylon-66 membranes, the PVDF membrane evaporated at $60{ }^{\circ} \mathrm{C}$ showed a particulate structure (Fig. 3). The PVDF membranes prepared at high evaporation temperature, however, differed to a large extent (Fig. 4). Nylon-66 particles exhibited linear grain boundaries between neighboring particles. PVDF particles coalesced each other to form a dense-like membrane. Although the exact structure of polymer molecules within these particles is not yet satisfactorily resolved, the coalescence of PVDF particles reflects crystalline and amorphous phases are simultaneously present within the particles or at least a heterogeneity region exists at the particle interface. As solvent leaves from the casting solution, the particle centers approach each other. When two particles are in contact, the amorphous phase supplies sufficient chain mobility through a reptation mechanism to reorganize the membrane structure [16], which is interrupted at the time of polymer vitrification. This suggests that microcrystallite formation with participation of the amorphous fraction could be at the origin of the particulate morphology. If the growth of particles is dominated by crystallization only, these particles should not be driven together to disappear the boundary. A typical example of this type is the Nylon-66 membranes. In this case, growth of particles should be so rapid to hold its integrity since the low boiling point of formic acid used as the solvent has a very rapid evaporation rate. Thus, the rate of polymer crystallization in solution is an important factor in controlling membrane structure.

\section{Conclusion}

The effect of evaporation temperature on the structures of the crystalline polymer membranes prepared by dry-cast process was studied. Although the dry process is a simple experimental method, it was found that the slight temperature change could affect the membrane morphology to a great degree. We offered a qualitative model of the nucleation and growth mechanism for membrane formation, which is consistent with both the SEM observation and DSC analysis on the membranes. The principal structure-determining factor is the rate of polymer crystallization during the solvent evaporation accompanied by the increased polymer concentration, which lead to a high viscous state to restrain chain mobility with a preferred orientation. This work shows that the membranes prepared by dry-cast process with a suitable evaporation temperature provide a particulate morphology and particles with a narrow size distribution.

\section{Acknowledgements}

The authors thank the National Science Council of the Republic of China for their financial support of project NSC 89-2216-E-002-006.

\section{References}

[1] Kesting RE. Synthetic polymeric membranes. New York: Wiley; 1985.

[2] Wijmans JG, Rutten HJJ, Smolders CA. Phase separation phenomena in solutions of poly(2,6-dimethyl-1,4-phenyleneoxide) in mixtures of trichloroethylene, 1-octanol, and methanol: relationship to membrane formation. J Polym Sci Polym Phys 1985;23:1941-55.

[3] Young TH, Lin DT, Chen LY, Huang YH, Chiu WY. Membranes with a particulate morphology prepared by a dry-wet casting process. Polymer 1999;40:5257-64.

[4] Mulder M. Basic principles of membrane technology. Dordrecht, The Netherlands: Kluwer; 1991.

[5] Castellari C, Ottani S. Preparation of reverse osmosis membranes. A numerical analysis of asymmetric membrane formation by solvent evaporation from cellulose acetate casting solutions. J Membr Sci 1981;9:29-41.

[6] Zeman L, Fraser T. Formation of air-cast cellulose acetate membranes. Part II: kinetics of demixing and macrovoid growth. J Membr Sci 1994;87:267-79.

[7] Huang RYM, Feng X. Studies on solvent evaporation and polymer precipitation pertinent to the formation of asymmetric polyetherimide membranes. J Appl Polym Sci 1995; 57:613-21.

[8] Lloyd DR, Kinzer KE, Tseng HS. Microporous membrane formation via thermally induced phase separation. I. solidliquid phase separation. J Membr Sci 1990;52:239-61.

[9] Bulte AMW, Folkers B, Mulder MHV, Smolders CA. Membranes of semicrystalline aliphatic polyamide nylon 4,6: formation by diffusion-induced phase separation. J Appl Polym Sci 1993;50:13-26.

[10] van de Witte P, Esselbrugge H, Dijkstra PJ, van de Berg JWA, Feijen J. A morphological study of membranes obtained from the systems polylactide-dioxane-methanol, polylactide-dioxane-water, and polylactide-N-methyl pyrrolidone-water. J Polym Sci Polym Phys 1996;34: 2569-78.

[11] Cheng LP, Dwan AW, Gryte CC. Membrane formation by isothermal precipitation in polyamide-formic acid-water systems. I. Description of membrane morphology. J Polym Sci Polym Phys 1995;33:211-22.

[12] Cheng LP, Dwan AW, Gryte CC. Membrane formation by isothermal precipitation in polyamide-formic acid-water systems. II. Precipitation dynamics. J Polym Sci Polym Phys 1995;33:223-35. 
[13] Cheng LP, Lin HY, Chen LW, Young TH. Solute rejection of dextran by EVAL membranes with asymmetric and particulate morphologies. Polymer 1998;39:2135-42.

[14] Lin DT, Cheng LP, Kang YJ, Chen LW, Young TH. Effects of precipitation conditions on the membrane morphology and permeation characteristics. J Membr Sci 1998;140:185-94.
[15] Young TH, Yao CH, Sun JS, Lai CP, Chen LW. The effect of morphology variety of EVAL membranes on the behavior of myoblasts in vitro. Biomaterials 1998;19:71724.

[16] Pinnau I, Koros WJ. A qualitative skin layer formation mechanism for membranes made by dry/wet phase inversion. J Polym Sci Polym Phys 1993;31:419-27. 